\title{
Parallel femtosecond laser processing with vector-wave control
}

\author{
Yoshio Hayasaki and Satoshi Hasegawa \\ Center for Optical Research and Education (CORE), Utsunomiya University \\ hayasaki@opt.utsunomiya-u.ac.jp
}

\begin{abstract}
Parallel femtosecond laser processing with a computer-generated hologram displayed on a spatial light modulator, has the advantages of high throughput and high energy-use efficiency. For further increase of the processing efficiency, we demonstrated parallel femtosecond laser processing with vector-wave control that is based on polarization control using a pair of spatial light modulators.
\end{abstract}

\section{Introduction}

A computer-generated hologram $(\mathrm{CGH})$ allows arbitrary control of spatial pulse shaping, and a spatial light modulator (SLM) displaying a dynamic CGH has been used to achieve variable spatial shaping of femtosecond laser pulses. Femtosecond laser processing with a CGH, called holographic femtosecond laser processing [1-3], has the advantages of high-throughput pulsed irradiation and high energy-use efficiency. In the holographic femtosecond laser processing previously reported, a simple linear or circular polarization was used. In order to enhance the photon-matter interaction with dependent on the property of material in laser processing, polarization control is indispensable.

In this paper, we propose a novel laser processing method based on polarization control of parallel pulses. The laser processing system is composed of a pair of SLMs displaying a dynamic CGH, one for making parallel pulses from incoming pulse and the other for controlling the polarization of the diffraction pulse. We demonstrate parallel processing with the different polarization simultaneously. We call this method the vector-wave femtosecond laser processing because of a use of the vector control of polarization.

\section{Principle of vector control of laser pulses}

When Jones matrices of two SLMs, a half-wave plate (HWP), and a quarter-wave plate (QWP) are given as $S \alpha, S \beta$, $H$, and $Q$, the electric field of the output beam $E_{\text {out }}$ for electric field of an input beam $E_{\text {in }}$ after passing through the optical devices is denoted as,

$$
E_{\text {out }}=Q_{\frac{\pi}{4}} S_{\beta} H_{\frac{\pi}{8}} S_{\alpha}=\sqrt{2}(1+i) \exp (i \alpha)\left[\begin{array}{c}
\cos \frac{\beta}{2}+\sin \frac{\beta}{2} \\
\cos \frac{\beta}{2}-\sin \frac{\beta}{2}
\end{array}\right] E_{\text {in }}
$$

where the subscripts of $Q$ and $H$ are the orientations of HWP and QWP, and $\alpha$ and $\beta$ are the spatial distributions of the phase modulation on an SLM, respectively. For the sake of the simplicity, the description of coordinate was omitted. Figure 1 shows the examples of the polarization control using SLMs.

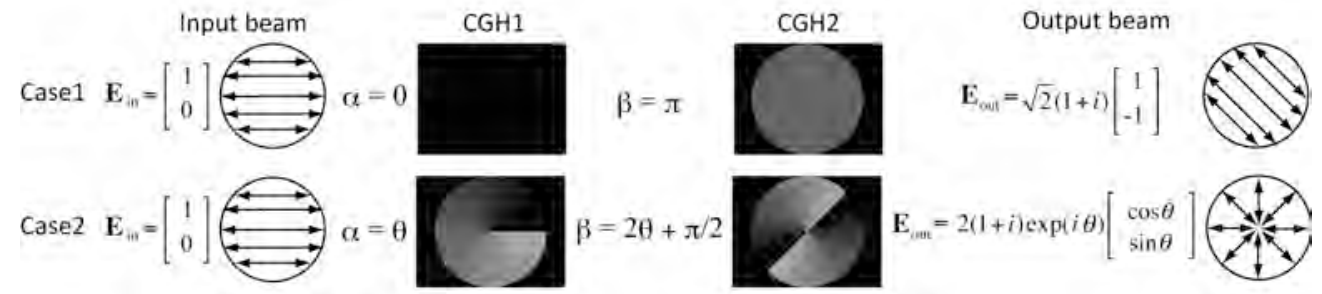

Fig. 1 Vector control of polarization.

\section{Experimental setup}

Figure 2 shows the vector-wave femtosecond laser processing system mainly composed of an amplified femtosecond laser system, liquid crystal on silicon SLMs (LCOS-SLMs, Hamamatsu), a relay optics, and a personal computer (PC). The femtosecond pulses from the laser system had a center wavelength $\lambda_{\mathrm{c}}=800 \mathrm{~nm}$, a spectral width $\Delta \lambda=8 \mathrm{~nm}$ (full width at half-maximum, FWHM), a repetition of $1 \mathrm{kHz}$. The pulse width was $\sim 110 \mathrm{fs}$ at the sample plane. The pulse was firstly irradiated to the SLM1 displaying the CGH1 for generating the parallel pulses in 
addition with a phase modulation required for the polarization modulation. The spatially-modulated pulse was image on the SLM2 displaying the CGH2. The spatially and polarization modulated wave was irradiated to a sample through the laser processing optics including an objective lens $(\mathrm{OL})$ (numerical aperture, NA $=0.85$ ). The sample was observed with an illumination of a halogen lamp (HL) and a charge coupled device (CCD) image sensor through a dichroic mirror (DM) and an infrared (IR) cut filter. The irradiation energy $E$ was measured at the sample plane.

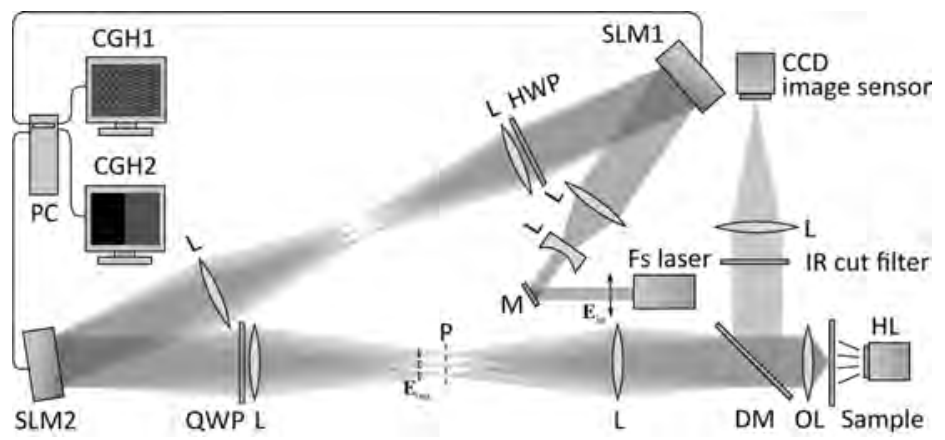

Fig. 2 Vector-wave femtosecond laser processing based on polarization control using a pair of spatial light modulators.

\section{Experimental results}

Figure 3 shows parallel femtosecond laser pulses with vector-polarization control. The arrows indicate the polarization states. The reconstructions were obtained by a cooled CCD image sensor on the 1st Fourier plane indicated by $\mathrm{P}$. The space between the parallel beams was $1.6 \times d_{\text {Airy }}$, where $d_{\text {Airy }}$, is the Airy disk diameter of $122 \mu \mathrm{m}$. Figure 3(a) shows the reconstruction of the parallel beams without the vector polarization control. The parallel beams having the same linear polarization had disturbances. The polarization control such that the neighboring beams had the cross polarization had few disturbances as shown in Fig. 3(b). Figures 3 shows the polarization controlled parallel beams through an analyzer with two orthogonal directions. The orthogonal polarizations in the parallel beams were confirmed.

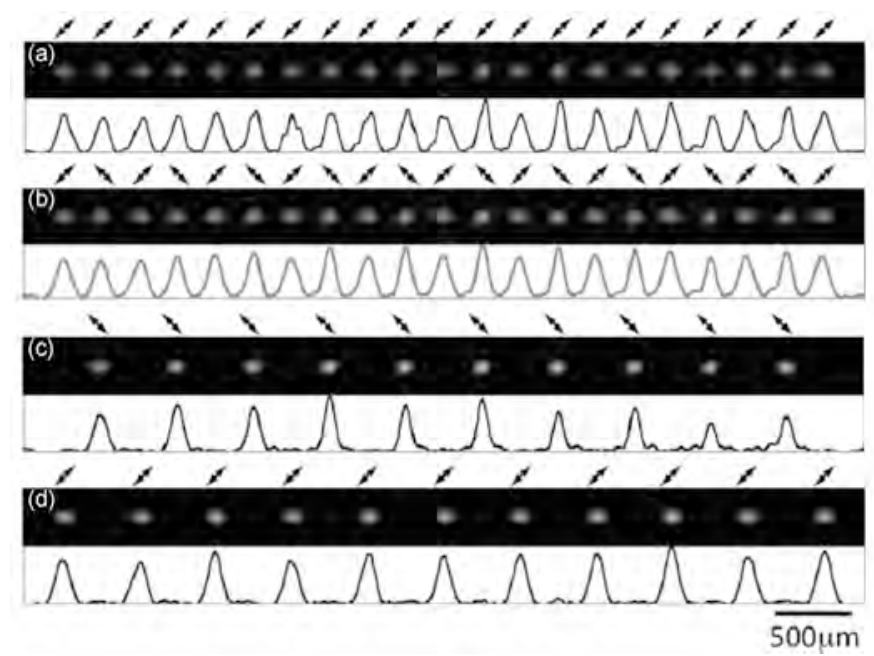

Fig. 3 Reconstructions (a) without and (b) with the vector polarization control. (c) and (d) Reconstruction through an analyzer with the respective orthogonal directions.

\section{Summary}

We proposed a novel method implemented with a pair of SLMs that performed vector wave control based on polarization control of laser pulses in parallel femtosecond laser processing.

[1] Y. Hayasaki, T. Sugimoto, A. Takita, and N. Nishida, "Variable holographic femtosecond laser processing by use of a spatial light modulator," Appl. Phys. Lett. 87, 031101 (2005).

[2] S. Hasegawa, Y. Hayasaki, and N. Nishida, "Holographic femtosecond laser processing with multiplexed phase Fresnel lenses," Opt. Lett. 31, 1705-1707 (2006).

[3] S. Hasegawa and Yoshio Hayasaki, "Second harmonic optimization of computer-generated hologram," Opt. Lett. 36, 2943-2945 (2011). 